\title{
CPCRT: Crosslayered and Power Conserved Routing Topology for congestion Control in mobile ad hoc networks
}

\author{
V. V. Appaji ${ }^{1}$, Dr. Sreedhar ${ }^{2}$ \\ ${ }^{I}$ (v.v. Appaji is currently working as associate professor at the Sphoorthy Engineering College, Hyderabad, and \\ doing his PhD at Bharath university, chennai, India, \\ ${ }_{2}^{2}$ Dr.M.Sridhar is currently working as Director R\&D, Bharath University, Chennai, India,
}

\begin{abstract}
All nodes in a Mobile Ad hoc Network are having mobility and dynamically tied in a subjective approach. Due moving directions caused by mobility, the frequent link failure happens, which consequences in packet losses. The protocol used in transmission handling in general fails to identify the root cause of the packet dropping hence assumes that these packet losses are due to congestion only. This wrong assumption need packet retransmissions till packet arrive successfully at the receiver. The protocols used in mobile ad-hoc networks are based on the layered architecture. The layered approach is extremely rigid and strict since each layer of the architecture is only concerned about the layers immediately above it or below it. Current wireless protocols rely on significant interactions between various layers of the network stack. In this context in our earlier work we proposed a crosslayered routing topology in short CRT to improve the congestion detection and handling strategy. The CRT is Mac, application and physical layer centric in particular. With the motivation gained from $C R T$, here in this paper we propose a crosslayered power conserved routing topology (CPCRT) for mobile ad hoc networks. The goal is to improve transmission performance by distinguishing between packet loss due to link failure and arbitrary loss of packets along with power conservation that used for packet transmission. Hence in our proposed cross layer routing topology the strength of the communication signal between hop level nodes is used to determine link failure. The objective of the CPCRT is to distinguish between packet loss due to link failure and arbitrary loss of packets and ensure QOS at the application layer along with conserving power that used at node level to transmit packets. The experiment results emerged as an evidence for better resource utilization and power conservation in congestion controlling by proposing crosslayered and power conserved routing topology.
\end{abstract}

Keywords: Manet; routing protocol; congestion control; cross layer; CRT, CPCRT, Mac, Mobile ad hoc network routing, $T C P$

\section{Introduction:}

While the TCP congestion handling is highly efficient to work over the Internet, mobile ad hoc networks displays some exceptional properties that generally affect the design of the appropriate protocols and protocol stacks in a substantial manner and of a congestion handling mechanism in meticulous. The huge ecological disparities in a mobile ad hoc network pose huge problems for standard TCP.

The node mobility and a shared, wireless multi-hop channel are the principal properties of MANETs. Vary in routes is indicative of node mobility and of the intrinsically unpredictable medium which results in unsteady packet delivery delays and packet losses which are not to be construed as congestion losses.

Using a wireless multi-hop channel permits a single data transmission only within the interference range of one node. Hence, physical close links are dependent on one another thereby influencing the manner in which the network congestion largely manifests itself. A distinctive Internet router is a dedicated host that is connected by high bandwidth links. Whenever there is Internet jams enchanting place, it is usually focused on one single router. On the contrary, MANET congestions affect the entire area due to a shared medium where regions of network and nodes are congested .

Packet losses, which normally depend on the network category, that are not owing to network congestions can be found to happen more often in wireless networks. These results in negative response of TCP congestion control. The watching of packet losses is very hard as the transmission times (as well as the round trip times) show a high difference.

A single sender is accidentally or purposely able of causing a network collapse due to congestion owing to the relatively low bandwidth of mobile ad-hoc networks. Severe inequity can take place among flows due to the severe result of a single traffic flow on the network situation. Traditional wire line networks similar to the Internet are not so prone to congestion-related problems as compared to wireless multi hop networks. We, therefore terminate that a balanced congestion handling is the foundation for network stability and superior performance . 
Because of the heterogeneous nature of application scenarios for multihop wireless networks, suitable congestion handling solutions for a specific network and application will mostly depend on the properties and the function of the relevant network [17]. Hence, there would be customized solutions for dissimilar scenarios in its place of a single, general purpose one as reflected in this paper. A majority of these proposals does not signify complete, ready-to-use protocols, but rather solutions for a subset of the recognized problems. These can serve as the basis for application-tailored protocol stacks. A small number of of the protocol possessions are, however, significant for a broader range of applications.

The past couple of years has seen the reception of wide focus on the problem of congestion handling both in the Internet circumstance, in accumulation to an ad-hoc network context. Much of the study focus has been on modeling, analysis, algorithm progress of end-to-end handling schemes (such as TCP), and adaptation of such schemes for ad-hoc networks. Algorithms that unite and steady operations have been industrious, given the routing path and the bandwidth constraints. However, in the context of a wireless network, another main constraint is due to the MAC (Media Access Control) layer [17]. Most wireless MACs utilizes a time-division strategy for accessing channel where at any point in space; the physical channel can be accessed by a single user at each moment of time (a time constraint).

The rest of the paper organized as in section 2 we explored the works most frequently cited in literature. Section 3 elaborates our earlier projected protocol CRT[18] and the proposed CPCRT. Section 4 reveals the simulations and their results, that followed by conclusions and references.

\section{Related Work:}

Congestion awareness and handling in networks is the issue that attains reasonable attention in from researchers. QoS centric congestion handling solution can be established in [1A]. Metrics based solution for congestion aware routing was proposed in [4]. Et al., [2] brings in metrics to assess data-rate, MAC overhead and buffer delay, which assist to identify and deal the congestion contention area in the network. Hongqiang Zhai, et al., [3] Proposed a solution by arguing that congestion and severe medium contention is consistent. Yung Yi et al., [4] Proposed a hop level congestion handling model. Tom Goff, Nael et al., [5] Discussed a set of algorithms that initiates alternative path usage when the quality of a path in use becomes thin. Xuyang et al., [6] present a cross-layer hop-by-hop jamming handling scheme designed to develop TCP performance in multihop wireless networks. Dzmitry et al [7] present the collision congestion on transport layer that mortify the performance. Duc et al., [8] argued that present designs for routing are not congested-adaptive.

Most of the existing models are aimed to identify the congestion through packet loss in routing path. The fair amount of times this packet loss can be an impact of link failure. Hence an attempt to packet outlet regularization to handling the packet loss that occurs against link failure is a useless attempt. The other expensive approach that opted by most of the existing solutions is regularizing the packet outlet at all nodes participating in routing. Most of the times it is possible to handling the congestion at hop node level [4] [15]. Hence packet outlet regularization at each node of the network would be an expensive in resource use. Here in this paper we argue that it is an essential requirement to identify the reason for packet loss. Hence we can avoid the congestion handling process via packet outlet regularization against link failure circumstances. And also we continue the argument that hop level congestion handling is not sufficient, because the when hop level nodes are not able to regularize the packet outlet load to handling the congestion, the resource utilization remains same as in source level packet outlet regularization models.

Here we propose a new cross layer routing topology for congestion handling, which considers

- The heterogeneity in node's packet inlet and outlet capacity

- Cross layered routing topology to distinguish between packet loss due to link failure and arbitrary packet loss.

\section{Crosslayered And Power Conserved Routing Topology (Crt) To Detect Congestion State In Routing}

\subsection{Crosslayered Routing Topology[18]}

In order to achieve desired objectives, there is a need for information flow among different layers in particular Mac, application and physical layers of the protocol stack which is termed as Crosslayered Routing Topology design (CRT) approach. It relies on the interactions between layers of the network stack.

The objective of the proposed cross layered mobile ad hoc network topology is to distinguish link failure and other root causes of congestion such as an overwhelmed packet inlet. In this context the transmission signal state that used for inlet packet at a node level, which determined at the physical layer helps to measure the link quality status. Hence the MAC layer communicates with physical layer to determine the signal state and then estimates link quality status. If signal state found to be good then estimates the possible interference and contention at nodes. If determined contention or transmission channel interference found to be high and causes congestion then Mac layer communicates with application layer to notify the congestion state. The link failure detection strategy of the CRT can be briefed as follows. 
1.2 Power conservation to achieve minimal Energy Usage

The nodes are having limited power and storage capacity, Hence the power conserved crosslayered routing topology has been proposed that saves power resources. Here in this proposed CPCRT model the RTS packet carries the transmission power used by the source node of that RTS. Then the target node of that RTS finds the state of the signal that used to transmit RTS.

$$
S S_{r}=S S_{s}(\alpha / 4 \pi d)^{2} S_{T} S_{R}
$$

Here $a$ is the wavelength of the carrier signal, $d$ is the distance traveled by RTS between source and target nodes. $S T$ is the omnidirectional transmission threshold of source node antennas and $S R$ is the omnidirectional receiving threshold of target node antennas. ' $S S_{s}$ ' is the actual state of the transmission signal power at the source node $s$. And $S S_{r}$ is the state of the signal power that found at target node $r$, which used to transmit RTS .

Then the loss state of signal $S S_{l}$ during routing can be found at target node $r$ by using the following equation.

$$
S S_{l}=S S_{r}-S S_{s}
$$

And then this $S S_{l}$ can be used to find minimal signal state $S S_{m}$ required at the source node, the equation is as follows

$$
S S_{m}=m h \times\left(S S_{l}+R S S_{m}\right)
$$

Here in the above equation

The ' $S S_{m}$ ' indicates minimal signal state required at the source node $s$

The ' $m h$ ' is the marginal hike threshold that is used to normalize $S S_{m}$ to handle the inference issues on the target node side.

The ' $R S S_{m}$ ' indicates the minimal signal state required at receiving node side to detect the appropriate signal.

There are a set of protocols available for power control in mobile ad-hoc networks based on the common power approach [14]. These protocols are complex and have been analyzed that the variable range transmission power is a better approach than the general power.

The proposed CPCRT is capable to conserve the power even to transmit RTS/CTS packets, which is based on the received signal state. When a source node wants to transmit data, it initiates the optimal routing strategy such as AODV and then broadcasts the RREQ packet to the hop level nodes and the RREP packet is received from the intermediate nodes via the shortest route and then enters it in their routing table about the next hop to which the anon data packets are desired to be advanced.

For power preservation, the RREP packet is recognized by an identifier (id) at the MAC layer and its signal state information is attained from the physical layer. Upon receiving the RREP packet by a node ' $r$ ' from a node ' $s$ ', the node ' $r$ ' computes loss state of the signal $S S_{l}$ during the RREP transmission from node ' $s$ ' to ' $r$ ' and minimal signal state $S S_{m}$ required at node ' $S$ '. And then node ' $r$ ' stores minimal signal state required for the node ' $S$ ' in its routing table.

The process of the proposed CPCRT as follows:

The source node ' $s$ ', while sending $R T S$ to its next hop level node $r$ of the routing path, also sends the $S S_{m}(r)$ stored in its routing table. Here $S S_{m}(r)$ is the minimal signal state required for $r$, which is measured and stored in the routing table of node $s$ during route discovery. The source node $s$ also includes $S S_{m}(s)$ as an extra field in the RTS packet. Upon receiving the RTS, the target node $r$ tunes its transmission power and replies back with ' $C T S$ ' packet. Upon receiving the $C T S$ the source node $S$ sends the data with the requisite transmission power informed by the target node $r$ through ' $C T S$ '.

\section{Determining The Congestion Status[18]}

The state of the transmission signal used to receive a packet can be estimated at the physical layer of the topology. This information is transferred to the MAC layer along with the signal state information. Then The MAC layer founds link quality status by using signal state information received from from the physical layer and then the determined link quality status will be intimated to routing layer by using a routing control packet. The link quality status information received from Mac layer will stored in the routing table by routing layer and 
it is used in some decision making process. The IEEE 802.11 is a consistent MAC protocol and it assumes permanent highest transmission, since RTS has to reach each exposed node and every CTS must reach every hidden node to avoid collision. In a gist the advantage of this process has been that unproductive retransmissions against packet drops caused by link failures can be avoided.

\section{Congestion Control Strategy In Corsslayered Routing Topology (Crt)}

The packet dropping is a very frequent and unavoidable situation in Manets. The reasons for this packet reducing can be classified as

- Transmission Link failure.

- Inferred Transmission due to overwhelming Packet inlet that leads Packet inlet receiving strength to short.

This also can maintain as packet reducing due to congestion at routing.

The nodes participating in the routing path discovered will be partitioned into groups, and then the status of congestion will be handled in two stages

- The Status of congestion within the group

- The status of congestion between the groups

This helps to minimize the packet outlet streamlining costs.

\subsection{Preparing node groups those participating in routing path}

The nodes participating in the selected routing path will be grouped based on their packet throughput status. In the process of root response the nodes will be grouped. The briefing of the process follows. In route response it group forming process will be initiated.

First a group $g_{i}$ with zero nodes will be considered.

The node that visited by response packet will be added to the group $g_{i}$ and then the average throughput $a t\left(g_{i}\right)$ of the nodes that are grouped will be measured. If $a t\left(g_{i}\right) \geq \zeta_{g}$ (here ' $\zeta_{g}$ ' is group level throughput threshold ) then concludes a group and start preparing a new group with nodes visiting thereafter. In this process one or more groups will be generated and each group contains one or more nodes such that the average throughput $a t(g)$ is greater or equal to the group level throughput threshold $\zeta_{g}$. During the process of group formation the degree of inlet strength for each group will be measured. This can achieved by finding the average of the degree of inlet capacity of nodes of that group. Let consider ' $\operatorname{dol}_{i}$ ' is the degree of the packet outlet at the node $i$. Then the degree of outlet at a group $g(i)$ of node $i$ can be measured as follows.

$d o l_{g(i)}=\frac{\sum_{i=1}^{|g(i)|} d o l_{i}}{|g(i)|}$

\subsection{Congestion State prediction algorithm(CSPA)}

Congestion State Prediction Algorithm in short can refer as CSPA explored in this section. CSPA is an optimal algorithm that helps to find the state of the packet dropping under congestion. This evaluation occurs under the Mac layer with the support of physical layer and then alerts application layer.

\subsubsection{Description of the notations used in CSPA algorithm \\ $\zeta_{T} \quad$ Predefined threshold that represents an interval \\ between two transmissions at one hop level \\ $\zeta_{t} \quad$ Actual period between last two transmissions \\ $\zeta_{\text {et }} \quad$ Elapsed time while last broadcast at one hop level \\ $I R S_{\zeta_{T}} \quad$ Average packet inlet receiving strength threshold \\ observed for predefined interval $\zeta_{T}$ \\ $\partial^{\prime} \quad$ Average threshold of the receiving strength \\ $I_{\text {Ce }} \quad$ Expected packet inlet receiving strength threshold \\ at current interval \\ IRS $\quad$ Packet Inlet receiving strength ratio \\ IRS $\quad$ Current packet inlet receiving strength ratio}




\subsubsection{CSPA for determining root cause of congestion}

At an event of packet receiving by node $i$ :

Physical layer determines the state of the communication signal that used to receive a packet as follows

if $\left(\zeta_{t}<\zeta_{T}\right) d o$

$\delta^{\prime}:=\frac{1}{2}\left(\frac{I R S_{c r}-I R S_{\zeta_{T}}}{\zeta_{t}}\right)+\frac{1}{2}\left(\delta^{\prime}\right)$

$I R S_{\zeta_{T}}:=I R S_{c r}\left(\frac{\zeta_{t}}{\zeta_{T}}\right)+I R S_{\zeta_{T}}\left(\frac{\zeta_{T}-\zeta_{t}}{\zeta_{T}}\right)$

endif

if $\left(\left(\zeta_{t}\right) \neg<\left(\zeta_{T}\right)\right) d o$

$\delta^{\prime}:=\frac{I R S_{c r}-I R S_{\zeta_{T}}}{\zeta_{t}}$

$I R S_{\zeta_{T}}:=I R S_{C r}$

endif

Physical Layer sends $\operatorname{IRS}_{\zeta T}$ to Mac Layer

Mac layer process to detect the root cause of packet dropping

$I R S_{c e}=I R S_{\zeta_{T}}+\delta^{\prime} \zeta_{\text {et }}$

if $\left(I R S_{c e}<I R S_{r}\right) d o$

macAlert to applicationlayer:link-failure

else

Mac layer communicates with application layer to detect channel inference and contention state

MacAlert to application layer : congestion

endif

\section{Group Outlet Directive Algorithm (Goda) For Congestion Control}

4.3 The description of the notations used in the algorithm

$N \quad$ Number of nodes in entire network

$|g(i)| \quad$ Number of nodes in a group $g$ such that $i \in g$

$g(c) \quad$ Current active group

$g(p) \quad$ Predecessor group of the ' $g(c)$ '

$g(f) \quad$ Successor group to ' $g(c)$ '

$g_{i} \quad i^{\text {th }}$ Group in the routing path

$g(n) \quad$ Group of the node ' $\mathrm{n}$ '

$W T_{n} \quad$ Buffering time at node $\mathrm{n}$

$d_{o l} \quad$ Degree of packet outlet load of group $g$

$d_{k} \quad$ Node level degree of outlet load, here $k$ is the node id

$d o l_{R P} \quad$ Degree of outlet load at entire routing path

$|G| \quad$ Total number of groups

This event occurs at the application layer if and only if Mac-layer alert indicates the congestion situation. Once the steering protocol [13] got an alert from the Mac layer about the congestion at a node $i$, it alerts the neighbor node that is the predecessor of contention node $i$ and referred as ' $s$ ' for further discussion. Hence $s$ assess it's $d o l_{s}$ by evaluating with $d o l_{g}$ of group $g(s)$ (group of the node $s$ ). If $d o l_{s}$ is greater than 

$d o l_{g(s)}$ and disparity between $d o l_{s}$ and $d o l_{g(s)}$ is greater than equal to packet inlet threshold $\varepsilon$ then node $s$ regularize the packet outlet load by increasing its packet waiting time at buffer $W T_{s}$ such that $d o l_{s} \geq d o l_{g(s)}+\varepsilon_{g(s)}$.

Here $\varepsilon$ can be measured by the following equation

$\varepsilon_{j}=\frac{\sum_{k=1}^{|g(j)|} d o l_{g(j)}-d o l_{k}}{|g(j)|}$

If node $s$ not able to regularize its packet inlet such that contention node $i$ prevents from congestion then $s$ alerts the other nodes those belongs to $g(s)$. Hence the nodes those are predecessors to the source node $s$ and belongs to a group $g(s)$ in a routing path attempt to normalize their degree of packet outlet load $\mathrm{dol}$ using the methodology discussed above in this section. Then all nodes update their ' $d o l$ ' and ' $d o l_{g(s)}$ ' will update and then verifies integrity of the $d o l_{g(s)}$ by comparing with a degree of the outlet of the entire routing path $d o l_{r p}$. ' $d o l_{g(c)} \geq d o l_{r p}+\bar{\varepsilon}$ ' Concludes that congestion at contention node handled by packet outlet regularization at current cell level. If $d o l_{g(c)}<d o l_{r p}+\bar{\varepsilon}$ then GODA will be initiated at $g(p)$, which is predecessor group to $g(c)$. The whole process of packet outlet regularization at group level discussed above can be referred as GODA(Group Outlet Directive Algorithm). Hence the nodes belong to $g(p)$ attempt to regularize their packet outlet load by using GODA. If $d o l_{g(p)} \geq d o l_{r p}+\bar{\varepsilon}$ is true then concludes that the congestion at contention group has been minimized or removed because of the packet outlet load regularization at the group $g(p)$, if false then GODA will be applied at the predecessor group to ' $g(p)$ '. This process continues either congestion removed at victim group or source group reached. Either the case once process completed then $d o l_{r p}$ will be updated.

\subsection{Group outlet directive Algorithm (GODA)}

The notations used in Algorithm:

i: Victim node of the congestion

s: sender connected to i.

$\left\{n_{u 1}, n_{u 2}, \ldots, n_{u k}\right\}$ : All nodes predecessors to ' $s$ '.

$\left\{n_{d 1}, n_{d 2}, \ldots, n_{d k}\right\}$ : All nodes successors to ' $s$ '.

$\{g(p-m), \ldots, g(p-1)\}: \mathrm{m}$ predecessor groups to $g(p)$ in the routing path, here ' $g(c) \cong g(s)$ ' since the current group is the group that contains source node 's' to victim node ' $i$ '.

$\{g(c), g(c+1), \ldots, g(p+m)\}$ : Set of successor groups to $g(p)$ in routing path, here $g(p+m)$ is a group that contains target node of the routing path

$\varepsilon$ : group level packet inlet threshold

$\bar{\varepsilon}$ : Routing path level Packet inlet threshold

Algorithm:

Against to congestion alert from Mac layer to application layer at the group $g(c)$ the following process activated at the application layer

$\varepsilon_{g(c)}=\frac{\mid \sum_{k=1}^{g(c) \mid} d i l_{g(c)}-d i l_{k}}{|g(c)|}$

The following process occurs at the source node $s$ to victim node $i$

If $d i l_{s}>d i l_{g(c)}$ and $d i l_{s}-d i l_{g(c)} \geq \varepsilon_{g(c)}$ begin

$W T_{s}+=w t$

Note: Value of packet weighting threshold wt should be decided such that $\operatorname{dol}_{s} \geq \operatorname{dol}_{g(c)}+\varepsilon_{g(c)}$ 
Return.

Endif else

$S$ sends alert to other all nodes belongs to the group $g(c)$ about contention node $i$.

$\left\{n_{u 1}, n_{u 2}, \ldots, n_{u k}\right\}$ Those belonging to group $g(c)$ updates their $d o l$ and then $d o l_{g(c)}$ will be measured as

$d o l_{g(c)}=\frac{|g(c)|}{\sum_{k=1} d o l_{k}}$

If $d o l_{g(c)}>d o l_{R P}$ and $\left(d o l_{g(c)}-d o l_{R P}\right) \geq \bar{\varepsilon}$ begin

Alert: jamming at contention node switch at $g(c)$ level.

Return.

Endif else

GODA applies on each group g such that $g \in\{g(p-m), \ldots . . g(p-1)\}$ as follows

Foreach group $g \in\left\{Z_{d 1}, Z_{d 2}, \ldots, Z_{d m}, \ldots, Z_{T}\right\}$ begin

For each node $n$ belongs to $g$ begin

Measure $\mathrm{dol}_{n}$

End-of-for each

Measure $\mathrm{dol}_{g}$ as

$d o l_{g}=\frac{\sum_{k=1}^{|g|} d o l_{k}}{|g|}$

End-of-for each

$g(p-m)$ Measures $d o l_{R P}$ as

$d o l_{R P}=\frac{\sum_{i=1}^{|G|} d o l_{i}}{|G|}$

Hence source node $\mathrm{S}$ of the routing path that belongs to group $\mathrm{g}(\mathrm{p}-\mathrm{m})$, regularize it's packet outlet load.

End of else

End of else

\section{Simulations And Results Discussion}

In this section we look at the simulations conducted using Ns-2 simulator [16]. We carried out performance assessment using ns-2 with considerations described in table 2.

\begin{tabular}{|c|c|}
\hline No of Hops: & 225 \\
\hline Approximate Hop distance & 300 meters \\
\hline Approximate total network & $1000 \times 1000$ meters \\
\hline Approximate Cell Rdious & $100 \times 100$ meters \\
\hline Physical channel bandwidth & $2 \mathrm{mbps}$ \\
\hline Mac Layer: & $\begin{array}{l}802.11 \text { DCF with the option of } \\
\text { handshaking prier to data transferring }\end{array}$ \\
\hline Physical layer representation & 802:11B \\
\hline Performance Index & $\begin{array}{l}\text { Outlet directive cost and end-to-end } \\
\text { throughput }\end{array}$ \\
\hline Max simulation time & $150 \mathrm{sec}$ \\
\hline
\end{tabular}

Table 2: parameters used in NS-2 [16] for performance analysis

We carried out simulations on three dissimilar routes, which are diverse in length as the number of hops. Paths and their lengths are

- A path that contains 15 nodes

- A path contains 40 nodes 
- A path that contains 81 nodes

The same load given to all three paths with a standard interval of $10 \mathrm{Sec}$ load given in bytes can be originate in fig 4 . The fig 5 finish the throughput observed for the proposed CRT. The congestion control cost observed for CRT is in Fig 6.

The procedure of measuring jamming control fallows:

Based on the obtainable resources, bandwidth and liveliness, for each individual transaction a threshold value between 0 and 1 assigned. In the process of congestion evaluate and control the total cost was measured by summing the cost threshold of each event involved. In fig 8 we can find the comparison between congestion cost observed for CRT and congestion and contention control model [15].

$c c c=\sum_{e=1}^{E} c t_{e}$

Here $c c c$ is the cost of a congestion control, $E$ is the total number of events involved. $c t_{e}$ is cost threshold of an event $e$. The example events are" cost of communication between Mac, physical and application layers ", "alert from Mac to source node of victim node", "outlet directive cost of the participating groups", "packet inlet estimation and packet outlet directive". The figures 5 to 7 reveals the advantage of the CPCRT over any other cross layer congestion models such as [15], which is identical to the performance of CRT[18]. The figure 8 indicates the advantage of CPCRT over CRT in power conservation for data transmission

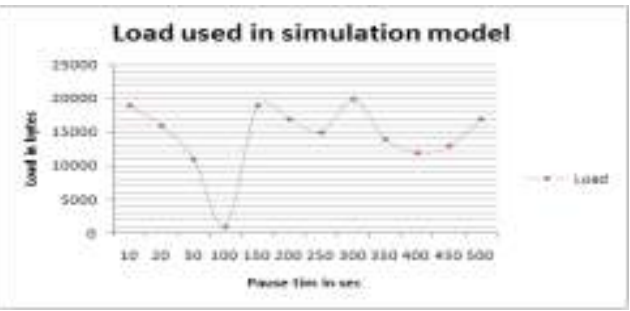

Fig 4: Data size in bytes is sent to destination node from the source node

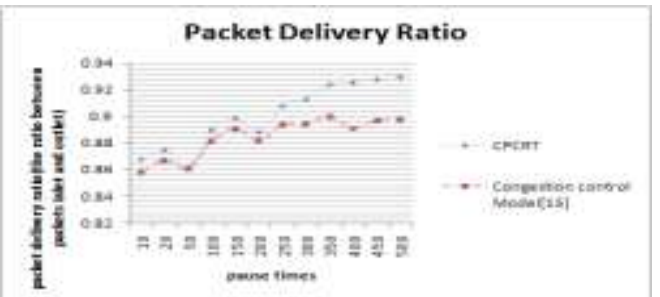

Fig 5: Throughput advantage of CPCRT over cross layer congestion control model [15]

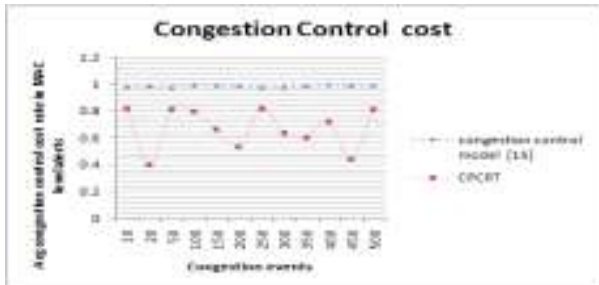

Fig 6: The advantage of CPCRT to minimize the cost to control congestion over cross-layered congestion control model [15]

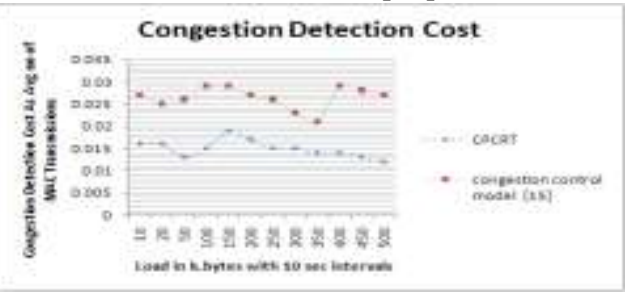

Fig 7: The advantage of CPCRT to minimize the cost to detect congestion over cross-layered congestion control model [15] 


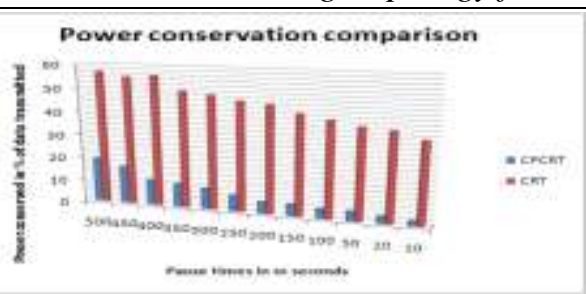

Fig 8: The Advantage of CPCRT over CRT in power conservation for data transmission

\section{Conclusion:}

A crosslayered and power conserved routing topology CPCRT is discussed in this paper. The CPCRT is an extension to our earlier invention called Crosslayered Routing Topology (CRT)[18].In CRT we introduced two algorithms Congestion State prediction algorithm(CSPA) and Group Outlet Directive Algorithm(GODA). CSPA helps to distinguish between packet loss due to link failure and arbitrary packet loss. Once the congestion contention node found, GODA attempts to resolve it at the source node to node identified as victim of congestion, if congestion not resolved at node level, attempts to handle at current group level, and the same continues at predecessor groups if failed to control congestion at current group level. Since the packet outlet directive is carried out at node level, current group level and all predecessor group levels in a succession, the cost of jamming control is minimal and required level. The same process has been used in CPCRT. In addition to that CPCRT introduced a cross layered power conservation routing topology. The required minimal state of the signal to transmit a data packet is identified during the route request and the same will be normalized during RTS/CTS. The simulation results that we observed are very impressive and promising. In particular, the proposed CPCRT is identical to CRT in all aspects but shown its advantage over CRT in power conserved data transmission(see figure 8). In future we can extend this work to tolerate the congestion by introducing path restoration strategy to current protocol CPCRT.

\section{References}

[1]. Michael Gerharz, Christian de Waal, and Matthias Frank, "A Practical View on Quality-of-Service Support in Wireless Ad Hoc Networks", BMBF.

[2]. Xiaoqin Chen, Haley M. Jones, A .D .S. Jayalath, "Congestion-Aware Routing Protocol for Mobile Ad Hoc Networks", IEEE, 2007.

[3]. Hongqiang Zhai, Xiang Chen, and Yuguang Fang, "Improving Transport Layer Performance in Multihop Ad Hoc Networks by Exploiting MAC Layer Information”, IEEE, 2007.

[4]. Yung Yi, and Sanjay Shakkottai, "Hop-by-Hop Congestion Control Over a Wireless Multi-Hop Network”, IEEE, 2007.

[5]. Tom Goff, Nael B. Abu-Ghazaleh, Dhananjay S. Phatak and Ridvan Kahvecioglu, "Preemptive Routing in Ad Hoc Networks", ACM, 2001.

[6]. Xuyang Wang and Dmitri Perkins, "Cross-layer Hop-byhop Congestion Control in Mobile Ad Hoc Networks", IEEE, 2008.

[7]. Dzmitry Kliazovich, Fabrizio Granelli, "Cross-layer Congestion Control in Ad hoc Wireless Networks," Elsevier, 2005.

[8]. Duc A. Tran and Harish Raghavendra, "Congestion Adaptive Routing in Mobile Ad Hoc Networks", 2006.

[9]. Nishant Gupta, Samir R. Das. Energy-Aware On-Demand Routing for Mobile Ad Hoc Networks, OPNET Technologies, Inc. 7255 Woodmont Avenue Bethesda, MD 20814 U.S.A., Computer Science Department SUNY at Stony Brook Stony Brook, NY 11794 4400 U.S.A.

[10]. Laura, Energy Consumption Model for performance analysis of routing protocols in MANET,Journal of mobile networks and application 2000.

[11]. LIXin MIAO Jian -song, A new traffic allocation algorithm in AD hoc networks, "The Journal of ChinaUniversity of Post and Telecommunication", Volume 13. Issue3. September 2006.

[12]. Chun-Yuan Chiu; Wu, E.H.-K.; Gen-Huey Chen; "A Reliable and Efficient MAC Layer Broadcast Protocol for Mobile Ad Hoc Networks," Vehicular Technology, IEEE Transactions on , vol.56, no.4, pp.2296-2305, July 2007

[13]. Giovanidis, A. Stanczak, S., Fraunhofer Inst. for Telecommun., Heinrich Hertz Inst., Berlin, Germany This paper appears in: 7th International Symposium on Modeling and Optimization in Mobile, Ad Hoc, and Wireless Networks, 2009. WiOPT 2009

[14]. Outay, F.; Vèque, V.; Bouallègue, R.; Inst. of Fundamental Electron., Univ. Paris-Sud 11, Orsay, France This paper appears in: 2010 IEEE 29th International Performance Computing and Communications Conference (IPCCC)

[15]. Yingqun Yu; Giannakis, G.B.; , "Cross-layer congestion and contention control for wireless ad hoc networks," Wireless Communications, IEEE Transactions on , vol.7, no.1, pp.37-42, Jan. 2008http://www.isi.edu/nsnam/ns/

[16]. Prof.K.Srinivas and Prof.A.A.Chari. Article: Cross Layer Congestion Control in MANETs and Current State of Art. International Journal of Computer Applications 29(6):28-35, September 2011. Published by Foundation of Computer Science, New York, USA

[17]. V. V. Appaji, Dr. Sreedhar; "Crosslayered Routing Topology for congestion control in mobile ad hoc networks"; International Journal of Scientific \& Engineering Research (ISSN 2229-5518) Volume 3,Issue 8(Paper got approved, publication pending) 